\title{
Study of X-ray Radiation Damage in Silicon Sensors
}

\author{
Jiaguo Zhang ${ }^{a *}$, Eckhart Fretwurst ${ }^{a}$, Robert Klanner ${ }^{a}$, Hanno Perrey ${ }^{a}$, loana \\ Pintilie $^{b}$, Thomas Poehlsen ${ }^{a}$, and Joern Schwandt ${ }^{a}$ \\ ${ }^{a}$ Institute for Experimental Physics, Hamburg University, \\ Luruper Chaussee 149, D-22761 Hamburg, Germany \\ ${ }^{b}$ National Institute of Materials Physics, \\ P.O.Box MG-7, Bucharest-Magurele, Romania \\ E-mail: jiaguo.zhang@desy.de
}

\begin{abstract}
The European X-ray Free Electron Laser (XFEL) will deliver 30,000 fully coherent, high brilliance X-ray pulses per second each with a duration below $100 \mathrm{fs}$. This will allow the recording of diffraction patterns of single complex molecules and the study of ultra-fast processes. Silicon pixel sensors will be used to record the diffraction images. In 3 years of operation the sensors will be exposed to doses of up to 1 GGy of $12 \mathrm{keV}$ X-rays. At this X-ray energy no bulk damage in silicon is expected. However fixed oxide charges in the insulating layer covering the silicon and interface traps at the $\mathrm{Si}-\mathrm{SiO}_{2}$ interface will be introduced by the irradiation and build up over time.
\end{abstract}

We have investigated the microscopic defects in test structures and the macroscopic electrical properties of segmented detectors as a function of the X-ray dose. From the test structures we determine the oxide charge density and the densities of interface traps as a function of dose. We find that both saturate (and even decrease) for doses between 10 and $100 \mathrm{MGy}$. For segmented sensors the defects introduced by the X-rays increase the full depletion voltage, the surface leakage current and the inter-pixel capacitance. We observe that an electron accumulation layer forms at the $\mathrm{Si}-\mathrm{SiO}_{2}$ interface. Its width increases with dose and decreases with applied bias voltage. Using TCAD simulations with the dose dependent parameters obtained from the test structures, we are able to reproduce the observed results. This allows us to optimize the sensor design for the XFEL requirements.

In addition the $\mathrm{Si}-\mathrm{SiO}_{2}$ interface region has been studied with time resolved signals induced by sub-nanosecond $660 \mathrm{~nm}$ laser light, which has a penetration of about $3 \mu \mathrm{m}$ in silicon. Depending on the biasing history, humidity and irradiation dose, losses of either electrons or holes or no charge losses are observed. The relevance of these results for the sensor stability and performance is under investigation.

KEYWORDS: XFEL; pixel sensors; surface radiation damage; simulations; charge losses.

\footnotetext{
${ }^{*}$ Corresponding author.
} 


\section{Contents}

1. Introduction 1

2. Irradiation with X-rays 2

2.1 Irradiation facility

2.2 Beam profile

2.3 Determination of the dose 3

3. Extraction of the concentrations of defects induced by X-rays from MOS capacitors

3.1 Method

3.1.1 Experimental techniques

3.1.2 Model calculation

3.2 Results 6

4. Influence on the performance of segmented sensors

4.1 Electrical properties 8

4.2 Charge losses 9

5. Summary and outlook 10

\section{Introduction}

Imaging experiments at the European X-ray Free Electron Laser (XFEL), planned to be operational in 2014, require silicon pixel sensors with extraordinary performance specifications: A dynamic range from single to $10^{5} 12 \mathrm{keV}$ photons deposited in less than $100 \mathrm{fs}$ in one pixel of $200 \mu \mathrm{m}$ $\times 200 \mu \mathrm{m}$, a time interval between XFEL pulses of $220 \mathrm{~ns}$, and radiation doses up to $1 \mathrm{GGy}$ for 3 years of operation. To optimize the sensor performance for these requirements, especially for radiation tolerance, demands an excellent understanding of the radiation damage caused by X-rays and its effects on segmented sensors.

The maximum energy transfer to silicon atoms from $12 \mathrm{keV} \mathrm{X}$-rays is $0.011 \mathrm{eV}$, which is far below the threshold energy of $21 \mathrm{eV}$ for bulk damage [1] and therefore no bulk damage is expected. $\mathrm{X}$-rays produce electron-hole pairs in the insulating layer (typically $\mathrm{SiO}_{2}$ or $\mathrm{SiO}_{2} / \mathrm{Si}_{3} \mathrm{~N}_{4}$ ). Some of these charge carriers produced by the $12 \mathrm{keV} \mathrm{X-rays} \mathrm{recombine,} \mathrm{whereas} \mathrm{those} \mathrm{remaining} \mathrm{either}$ remain in the insulating layer or move to the electrode on top of the insulating layer or drift to the $\mathrm{Si}-\mathrm{SiO}_{2}$ interface. Once holes come close to the $\mathrm{Si}_{-} \mathrm{SiO}_{2}$ interface a fraction of them will be trapped in the oxide close to the interface, and produce radiation induced fixed oxide charges. In addition, border traps [2] and interface traps are produced: The former are located in the oxide near the $\mathrm{Si}-\mathrm{SiO}_{2}$ interface and can be charged up or discharged through capture and emission 
of electrons and/or holes; whereas the latter are at the $\mathrm{Si}_{-} \mathrm{SiO}_{2}$ interface and have energy levels distributed throughout the silicon band gap and whose occupation therefore changes with band bending. They are responsible for the surface generation current. The densities of fixed oxide charges and interface traps introduced by X-rays mainly depend on the dose (energy deposition in the insulating layer), dose rate, electric field during irradiation, and post-irradiation conditions (e.g. time and temperature of annealing).

The fixed oxide charges and interface traps at the $\mathrm{Si}_{-} \mathrm{SiO}_{2}$ interface change the performance of segmented silicon sensors: This manifests itself as a shift in the full depletion voltage to larger values, an increase of the leakage current and the inter-pixel capacitance, a decrease of the interpixel resistance, and formation of an electron accumulation layer, which may cause charge losses at the interface. In this work we have irradiated MOS capacitors to determine the microscopic radiation damage parameters, and characterized the electrical properties of $\mathrm{p}^{+} \mathrm{n}$ silicon microstrip sensors irradiated to doses up to $100 \mathrm{MGy}$.

\section{Irradiation with X-rays}

\subsection{Irradiation facility}

For the irradiations, a facility has been set up [3] at the beam line F4 of HASYLAB DORIS III, DESY. The set-up is shown in figure 1. It consists of an adjustable Ta chopper, which permits the reduction of the full dose rate $(180 \mathrm{kGy} / \mathrm{s})$ down to $0.5 \%$, an adjustable collimator to precisely define the region of irradiation, and a sample holder with 5 biasing lines, which is connected to a liquid cooling system to control the temperature in the range of 10 to $30^{\circ} \mathrm{C}$.

The test structures and sensors were glued and wire-bonded to a ceramic substrate, shown at the top left of figure 1. This holder makes the exchange and test of the structures easy and safe.

The set-up is mounted on a table, which permits computer control of both the horizontal and vertical movement of the sample enabling larger areas than the beam spot to be irradiated uniformly. A pneumatic beam shutter, for a precise determination of the exposure time to the beam, was installed close to the exit window of the beam pipe.

\subsection{Beam profile}

The beam line F4 at DORIS III provides a "white" photon beam from a bending magnet. The energy spectrum was calculated taking into account the X-ray absorption by the materials in the beam line. As shown in figure 2(a) the typical photon energy at the detector was $12 \mathrm{keV}$ with the full width at half maximum of about $10 \mathrm{keV}$.

The profile of the X-ray beam was measured by the photocurrent in a silicon pad diode biased to $6 \mathrm{~V}$. The pad diode, glued onto a ceramic substrate as shown in figure 1, was placed onto the sample holder. The collimator was opened to a window of $0.2 \mathrm{~mm} \times 0.2 \mathrm{~mm}$. The sample was moved in steps of $0.1 \mathrm{~mm}$ to scan the beam. The photon flux as a function of position was calculated from the measured current, the average energy of $3.6 \mathrm{eV}$ to produce one electron-hole pair in silicon and the energy deposited by the photons. The fraction of interacting photons was derived from the energy spectrum and the absorption length of X-rays as a function of energy. Figure 2(b) shows the measured two dimensional beam profile. 


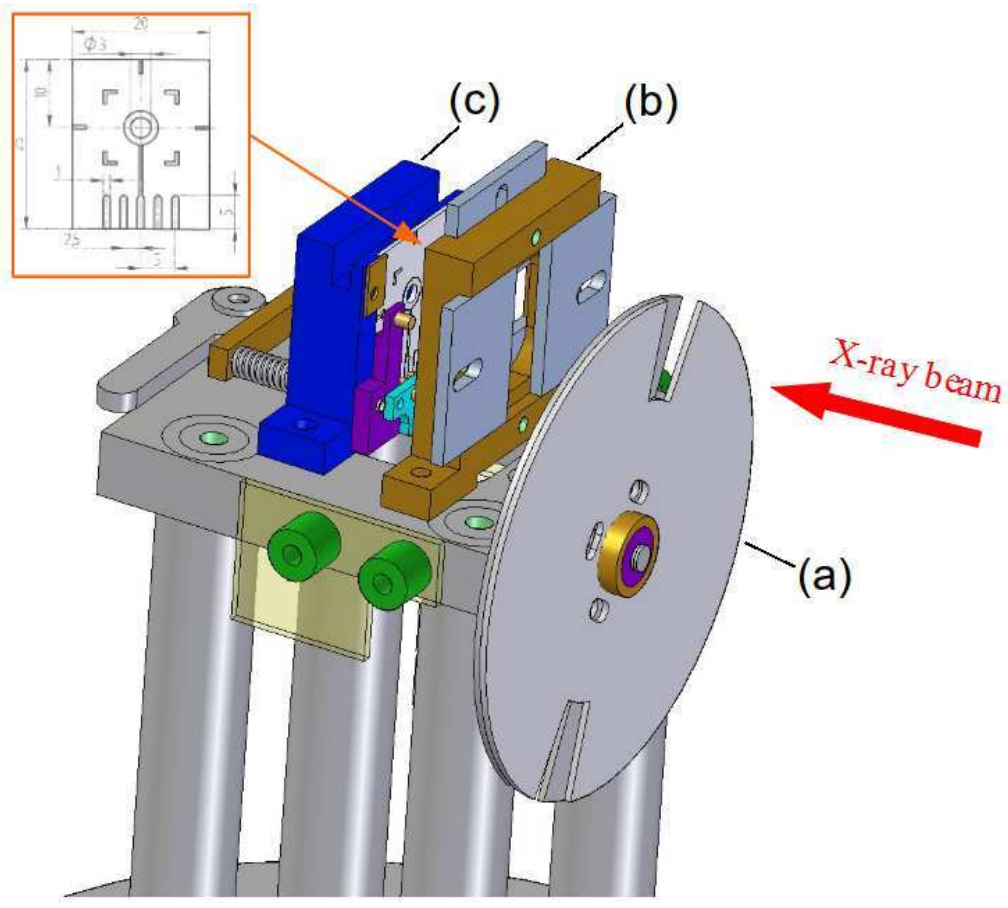

Figure 1. Set-up of the irradiation facility: Chopper (a), collimator (b), and sample holder (c) with cooling and spring mechanism. The ceramic substrate which is put into the sample holder carries test structures and sensors (shown at the top left). The entire set-up can be moved in all three dimensions by computer control.

The horizontal and vertical profiles at the center of the beam are shown in figure 2(c) and figure 2(d). The width of the horizontal profile is about $5 \mathrm{~mm}$ and uniform; whereas the full width of half maximum of the vertical profile is $4 \mathrm{~mm}$ and Gaussian-like. The distributions are as expected for the synchrotron radiation of the positrons of DORIS III in a bending magnet.

\subsection{Determination of the dose}

Using the data shown in figure 2(a) the normalised photon spectrum and figure 2(b) the two dimensional beam profile, the average dose in the insulator can be calculated according to:

$$
\text { Dose }=\frac{3.6 \mathrm{eV} \cdot R}{q_{0} \rho_{S i} d_{S i} A} \iiint_{\Delta x, \Delta y, t_{\text {irra }}} J_{\text {diode }}(x, y) \cdot d x d y d t
$$

with $J_{\text {diode }}(x, y)$ the current density $\left(A / \mathrm{mm}^{2}\right)$ measured by the silicon diode, $t_{\text {irra }}$ the duration of irradiation, $\rho_{S i}$ and $d_{S i}$ the density and thickness of the silicon diode. $A$ is the irradiated area of the detector, $\Delta x$ and $\Delta y$ the horizontal and vertical extensions of the detector in the plane perpendicular to the beam and $q_{0}$ is the elementary charge. $R$ is the ratio of energy deposited per unit mass in the insulator $\left(E_{\text {Insulator }}\right)$ to energy deposited in the silicon $\left(E_{S i}\right)$ for the given photon spectrum.

$$
R=\frac{\frac{E_{\text {Insulator }}}{\rho_{\text {Insulator }} \cdot d_{\text {Insulator }}}}{\frac{E_{S i}}{\rho_{S i} \cdot d_{S i}}}
$$

$\rho_{\text {Insulator }}$ and $d_{\text {Insulator }}$ are the density and thickness of the insulator. For the given energy spectrum, and $d_{\mathrm{SiO}_{2}}=300 \mathrm{~nm}$ and $d_{S i}=300 \mu \mathrm{m}$, the value of $R$ is 1.30 , which is essentially 

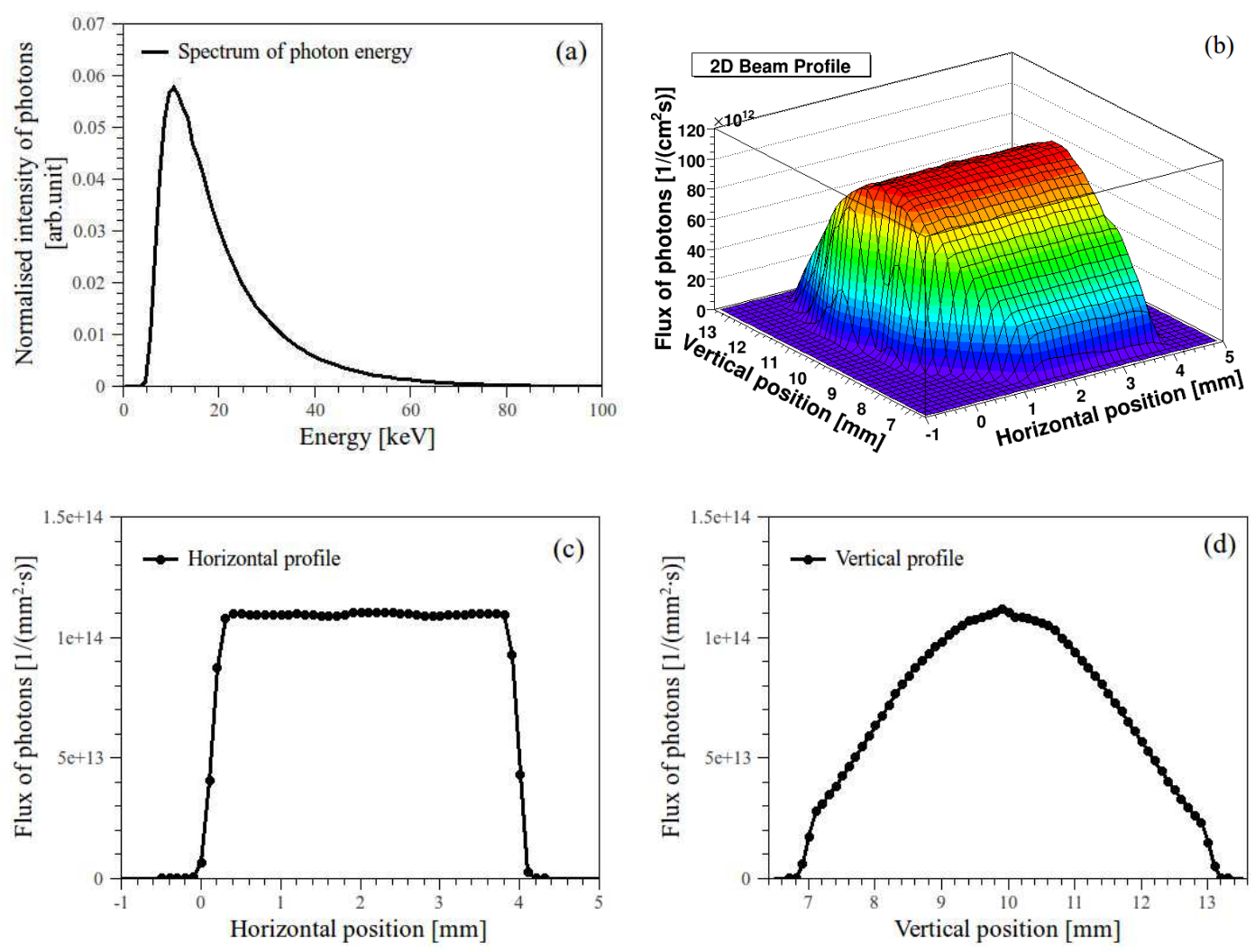

Figure 2. (a) Normalised spectrum of photon energy. (b) Two dimensional beam profile. (c) Horizontal profile. (d) Vertical profile.

independent of $d_{\mathrm{SiO}_{2}}$ for the typical oxide thickness of sensors. Without the chopper the typical dose rates in $\mathrm{SiO}_{2}$ are $180 \mathrm{kGy} / \mathrm{s}$, which corresponds to a flux of approximately $1.1 \times 10^{14}$ photons $/\left(\mathrm{mm}^{2}\right.$ $\cdot s)$.

The results from the measurement of the dose agree within $20 \%$ with the calculations using the current of the DORIS III beam, the field of the bending magnet and the geometry of the set-up. All references to dose in the text refer to the surface dose in $\mathrm{SiO}_{2}$. The dose enhancement in $\mathrm{SiO}_{2}$ has not been taken into account in this study [ [

\section{Extraction of the concentrations of defects induced by X-rays from MOS capaci- tors}

To study the radiation induced damage at the $\mathrm{Si}-\mathrm{SiO}_{2}$ interface, the Metal-Oxide-Semiconductor (MOS) capacitor was used. The MOS capacitors under study were fabricated by CiS on $280 \mu \mathrm{m}$ thick n-doped $<100\rangle$ substrates with a doping concentration of about $7.0 \times 10^{11} \mathrm{~cm}^{-3}$. The insulator is made of $350 \mathrm{~nm} \mathrm{SiO} 2$ covered by $50 \mathrm{~nm} \mathrm{Si}{ }_{3} \mathrm{~N}_{4}$. The diameter of the $\sim 1 \mu \mathrm{m}$ thick circular aluminium gate is $1.5 \mathrm{~mm}$.

The MOS capacitors were irradiated to $\mathrm{SiO}_{2}$ doses from $12 \mathrm{kGy}$ up to $1 \mathrm{GGy}$. The dose rate was $18 \mathrm{kGy} / \mathrm{s}$, except for the irradiation to $1 \mathrm{GGy}(180 \mathrm{kGy} / \mathrm{s})$ and to $12 \mathrm{kGy}(1.3 \mathrm{kGy} / \mathrm{s})$. The 
results obtained do not strongly depend on the dose rate according to our previous investigations [3].

\subsection{Method}

In a previous study of one highly irradiated MOS capacitor, it was found that one of the $\mathrm{Si}_{-} \mathrm{SiO}_{2}$ interface traps showed a fast annealing already at room temperature which made the measurements unreproducible. Therefore, in order to obtain reproducible results, all irradiated MOS capacitors were annealed for 10 minutes at $80{ }^{\circ} \mathrm{C}$ before the measurements.

\subsubsection{Experimental techniques}

The Capacitance-Voltage $(\mathrm{C}-\mathrm{V})$ and Conductance-Voltage $(\mathrm{G}-\mathrm{V})$ measurements have been performed at room temperature using an Agilent E4980A bridge. An AC voltage of $50 \mathrm{mV}$ and frequencies from $1 \mathrm{kHz}$ to $1 \mathrm{MHz}$ have been chosen. The voltage scan started at $0 \mathrm{~V}$ (strong accumulation in our case) and stopped before strong inversion was reached in order to avoid injecting holes into border traps [5].

In order to determine the parameters and the densities of interface traps the Thermally Dielectric Relaxation Current (TDRC) technique [6, 7] was employed. The TDRC technique is a well-established tool to study majority carrier traps at the $\mathrm{Si}_{-} \mathrm{SiO}_{2}$ interface of MOS capacitors. The TDRC measurement was carried out as follow: The MOS capacitor was cooled to low temperature (10 K using a helium cooling system) biased in strong accumulation to fill the interface traps with majority carriers (with electrons for n-type silicon). At $10 \mathrm{~K}$ the gate voltage was changed to bias the MOS capacitor in weak inversion, in which condition traps remained filled since the temperature was too low to allow the thermal emission of the electrons. Then the sample was heated up to room temperature with a constant rate of $\beta=0.183 \mathrm{~K} / \mathrm{s}$. As the temperature rises the trapped electrons are gradually released through thermal emission and the trap discharge current is recorded as TDRC signal. In general, the TDRC signal is due to both $\mathrm{Si}_{-} \mathrm{SiO}_{2}$ interface traps and traps in the depletion region of the bulk silicon. However, since the $12 \mathrm{keV} \mathrm{X}$-rays only introduce interface traps, the TDRC signal from bulk traps can be ignored.

Figure 3 shows the TDRC signal as a function of temperature for different doses after annealing at $80{ }^{\circ} \mathrm{C}$ for 10 minutes. A pronounced peak at $\sim 225 \mathrm{~K}$, corresponding to an energy of 0.60 $\mathrm{eV}$ as measured from the conduction band, and a broad distribution between $210 \mathrm{~K}$ and $50 \mathrm{~K}$ can be observed. The TDRC signal increases with dose up to $100 \mathrm{MGy}$ and decreases at $1 \mathrm{GGy}$. The decrease of the TDRC signal is probably due to the annealing of traps at high dose rates during the longer irradiation time.

In the analysis, we assumed that the interface states density of each trap are uniformly distributed in space with Gaussian-like energy distributions in the silicon band gap. Three Gaussian distributions were used to describe the measured TDRC spectra. The energy distribution and the density of interface states of each trap were calculated using the equations (16) and (17) in the paper of H. A. Mar [8] for separated, Gaussian distributed TDRC signals. For this calculation, electron capture cross sections for each trap are needed, which were obtained by minimizing the $\chi^{2}$ of the difference between the measured and calculated $\mathrm{C} / \mathrm{G}$ values for the frequencies $1,3,10$ and $30 \mathrm{kHz}$ at different gate voltages. The model calculation is discussed in the next section. Parameters, e.g. 


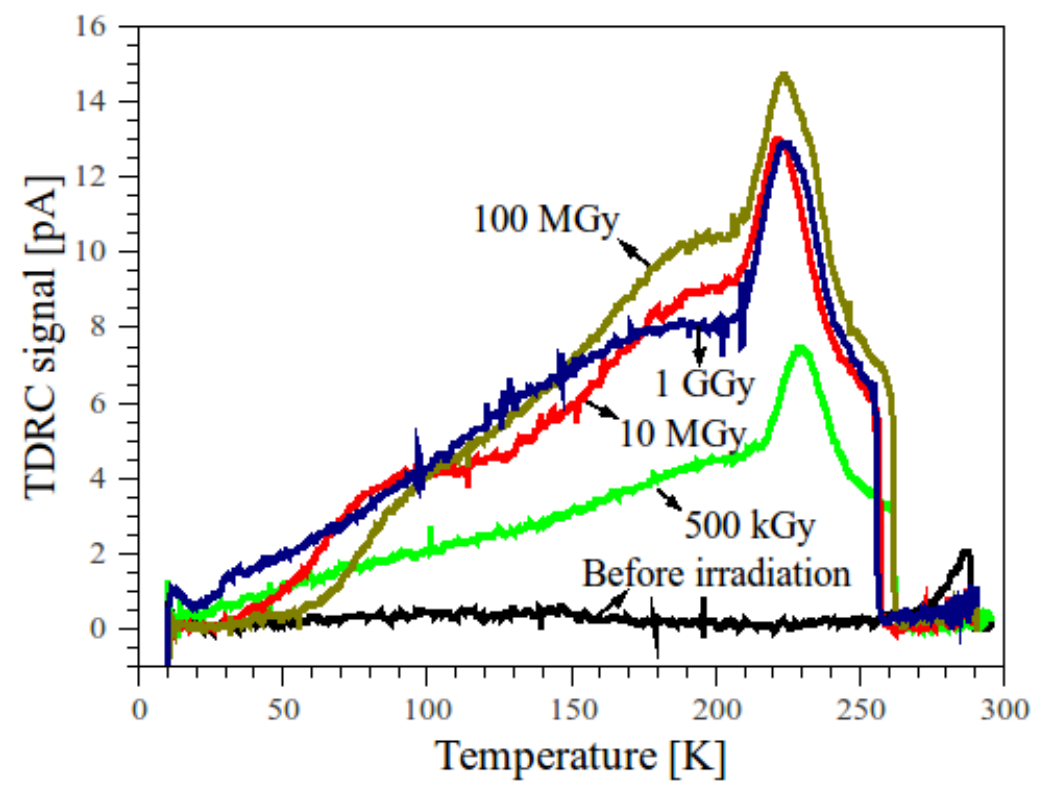

Figure 3. TDRC spectra $(\beta=0.183 \mathrm{~K} / \mathrm{s}$ ) of the MOS capacitors for different irradiations after annealing for 10 minutes at $80^{\circ} \mathrm{C}$. Only $0 \mathrm{kGy}$ (before irradiation), $500 \mathrm{kGy}, 10 \mathrm{MGy}, 100 \mathrm{MGy}$ and 1GGy are shown.

electron capture cross section, mean energy and full width of half maximum of each trap have been reported in [5].

\subsubsection{Model calculation}

The following model which includes the effects of the interface traps was used to describe the C/G-V measurements of the MOS capacitors. As shown in figure 4, the model consists of an RC circuit. The equations to calculate the capacitance and the resistance of each element in the circuit were described in [5]. The admittance of the circuit as a function of gate voltage was calculated based on the measured TDRC spectra, evaluated electron capture cross sections, doping concentration and fixed oxide charge density, and finally compared to the measured parallel capacitance and conductance. The doping concentration of the semiconductor close to the $\mathrm{Si}-\mathrm{SiO}_{2}$ interface was determined from the high frequency capacitance in strong inversion following the Lindner approximation [9]. The three interface traps were assumed to be acceptors. The fixed oxide charge density $N_{\text {ox }}$, which just causes a shift in voltage, were extracted from the voltage shifts observed in the measured $\mathrm{C} / \mathrm{G}-\mathrm{V}$ curves.

\subsection{Results}

It was found [5] that, after annealing at $80^{\circ} \mathrm{C}$ for 10 minutes, the fixed oxide charge density $N_{o x}$ and the interface trap densities $N_{i t}^{1,2,3}=\int D_{i t}^{1,2,3}\left(E_{t}\right) d E_{t}$ integrated over the band gap saturate at doses between 10 and $100 \mathrm{MGy}$. The saturation value of $N_{o x}$ and $N_{i t}^{1,2,3}$ are $2.8 \times 10^{12}, 1.2 \times 10^{12}, 0.7 \times 10^{12}$ and $0.6 \times 10^{12} \mathrm{~cm}^{-2}$, respectively. The extracted microscopic parameters related to the surface 


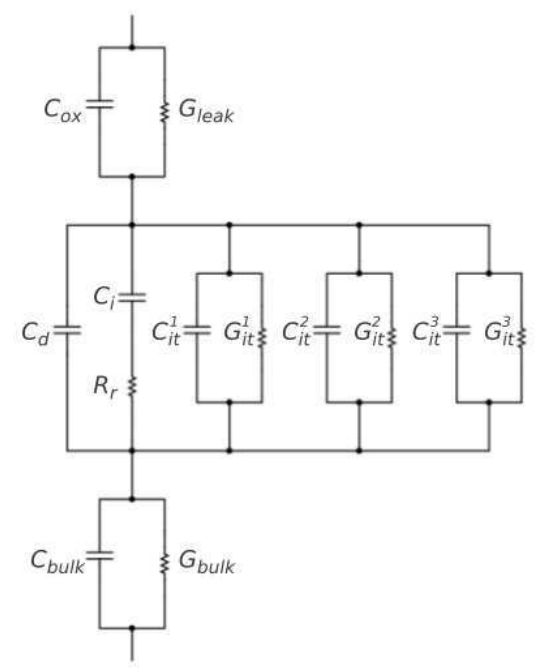

Figure 4. Model with three interface traps used to calculate the $\mathrm{C} / \mathrm{G}-\mathrm{V}$ curves for the MOS capacitors: $C_{\mathrm{ox}}$ and $G_{\text {leak }}$ is the capacitance and conductance of the insulator; $C_{\mathrm{d}}$, the capacitance of the depletion layer; $C_{\mathrm{i}}$, the inversion capacitance due to minority carriers accumulating at the interface; $R_{\mathrm{r}}$, the recombination/generation resistance; $C_{\mathrm{it}}^{\mathrm{i}}$ and $G_{\mathrm{it}}^{\mathrm{i}}$, the capacitances and conductances due to interface traps; $C_{\mathrm{bulk}}$ and $G_{\text {bulk }}$, the capacitance and conductance of the non-depleted silicon bulk. The relations between $C_{\mathrm{it}}^{\mathrm{i}}$ and $G_{\mathrm{it}}^{\mathrm{i}}$ and the microscopic properties of the traps are given in [5].

radiation damage caused by the X-rays have been implemented in Synopsys TCAD simulation [10] with the aim of optimizing the sensor design for the XFEL applications.

\section{Influence on the performance of segmented sensors}

To experimentally study the influence of the radiation induced damage on the performance of segmented sensors, we use AC-coupled $\mathrm{p}^{+} \mathrm{n}$ microstrip sensors. The microstrip sensors were fabricated by CiS on an n-doped silicon substrate with a thickness of $280 \mu \mathrm{m}$ and with the orientation $<100\rangle$. The doping concentration in bulk silicon is about $8.0 \times 10^{11} \mathrm{~cm}^{-3}$ (as determined from the $\mathrm{C}-\mathrm{V}$ curve of a close-by pad diode on the same wafer). The AC coupling between the $\mathrm{p}^{+}$implantation and the aluminium strips is made of $200 \mathrm{~nm} \mathrm{SiO}_{2}$ and $50 \mathrm{~nm} \mathrm{Si}_{3} \mathrm{~N}_{4}$. In between the aluminium

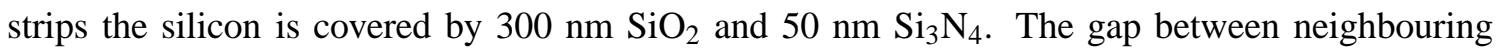
implants is $62 \mu \mathrm{m}$, the pitch $80 \mu \mathrm{m}$ and the length of the 98 strips of each sensor $7.9 \mathrm{~mm}$. All implanted strips are connected to a biasing ring through biasing resistors, which are made with a low dose $\mathrm{p}^{+}$implantation. The biasing ring, the current collection ring, namely the innermost ring close to the biasing ring, and 13 guard rings surround all strips and define the active detection area of the sensor.

The $\mathrm{p}^{+} \mathrm{n}$ microstrip sensors mounted onto the ceramic substrates as discussed in section 2.1, were irradiated to $\mathrm{SiO}_{2}$ doses of $1 \mathrm{MGy}, 10 \mathrm{MGy}$ and $100 \mathrm{MGy}$. After irradiation, in order to obtain reproducible results, the sensors were annealed at $80{ }^{\circ} \mathrm{C}$ for 60 minutes before the measurements. After the $100 \mathrm{MGy}$ irradiation the biasing resistors failed. As shown in figure 5(a), the biasing resistance, derived from the slope of the I-V measurement between one of the strips and the biasing 
ring, exceeds $100 \mathrm{M} \Omega$ after irradiation to $100 \mathrm{MGy}$. Whereas the values of the biasing resistor before irradiation, and after 1 and $10 \mathrm{MGy}$ are $0.5,0.6$ and $1.0 \mathrm{M} \Omega$. The failure of the biasing resistor can be explained by the removal of free holes in the low dose $\mathrm{p}^{+}$implants due to the positive oxide and interface charges. In the following we therefore will only present the results obtained for dose values of 0,1 and $10 \mathrm{MGy}$.

\subsection{Electrical properties}

The Capacitance-Voltage (C-V) and Current-Voltage (I-V) measurements have been performed at room temperature. For the measurement of the capacitance of the entire sensor, a DC power supply was connected to the rear side of the sensor through a $1 \mathrm{k} \Omega$ resistor; the high frequency terminal of the Agilent 4980A was connected to the rear side of the sensor through a capacitor of $1 \mu \mathrm{F}$ and the $\mathrm{AC}$ current from the biasing ring of the sensor fed back to the input terminal of the bridge. The current collection ring was grounded and the guard rings were kept floating during the measurement. Figure 5(b) shows the capacitance of the entire sensor measured at $100 \mathrm{kHz}$, plotted as $1 / \mathrm{C}^{2}$ versus bias voltage. $1 / \mathrm{C}^{2}$ increases linearly with bias voltage and saturates at the full depletion voltage $\mathrm{V}_{\text {dep }}$. A kink at $\sim 6 \mathrm{~V}$, the voltage at which the depletion regions below the individual strips merge, is observed. The full depletion capacitance $\mathrm{C}_{\mathrm{dep}}$ expected for a pad diode with the same area and thickness of the silicon substrate is $23.3 \mathrm{pF}$, close to the measured value of $23.6 \mathrm{pF}$ for the microstrip sensors. It should be noted that, compared to a pad diode, an additional voltage is needed for microstrip sensors to fully deplete the sensor. The full depletion voltage $V_{\text {dep }}$ of the microstrip sensor before irradiation is about $12 \mathrm{~V}$ larger than the value calculated from the same doping concentration and thickness for a pad diode. After irradiations, the full depletion voltage $\mathrm{V}_{\text {dep }}$ increases by an additional $\sim 10 \mathrm{~V}$, which is required to compensate for the positive charges in the oxide and at the $\mathrm{Si}-\mathrm{SiO}_{2}$ interface. The increase of the full depletion voltage $\mathrm{V}_{\text {dep }}$ saturates at high doses. Furthermore, the radiation introduced interface traps can be charged or discharged by an external AC signal, and thus act like a frequency dependent capacitor as seen in figure 5(c). This effect is implemented in the model for the MOS capacitor discussed in section 3.1.2.

The leakage current was measured from the biasing ring of the sensor by a Keithley 6517A electrometer. The biasing ring and the rear side of the sensor were connected to the current input terminal and the voltage output terminal of the electrometer, respectively. A Keithley 6485 picoammeter was used to monitor the current flowing into the current collection ring. All guard rings were left floating. The results of the measurements are shown in figure 5(d). Compared to the I-V curve without irradiation, the leakage currents after irradiations increase by two orders of magnitude and do not show any saturation with bias even above the full depletion voltage $\mathrm{V}_{\text {dep }}$. The leakage current is mainly due to the surface generation current from the $\mathrm{Si}_{-} \mathrm{SiO}_{2}$ interface regions not covered by an electron accumulation layer. The observed linear increase of the leakage current with bias voltage agrees with the results of the Synopsys TCAD simulation, which shows that the accumulation layer decreases approximately linearly with bias voltage. The measured leakage current agrees with the surface current of $\sim 8 \mu \mathrm{A} / \mathrm{cm}^{2}$ measured for highly irradiated gate controlled diodes [3].

Interstrip capacitance and interstrip resistance (not shown here), which are directly related to the electron accumulation layer at the $\mathrm{Si}-\mathrm{SiO}_{2}$ interface, increase with dose and decrease with 
applied bias voltage, as expected from the dose and voltage dependence of the width of the accumulation layer.
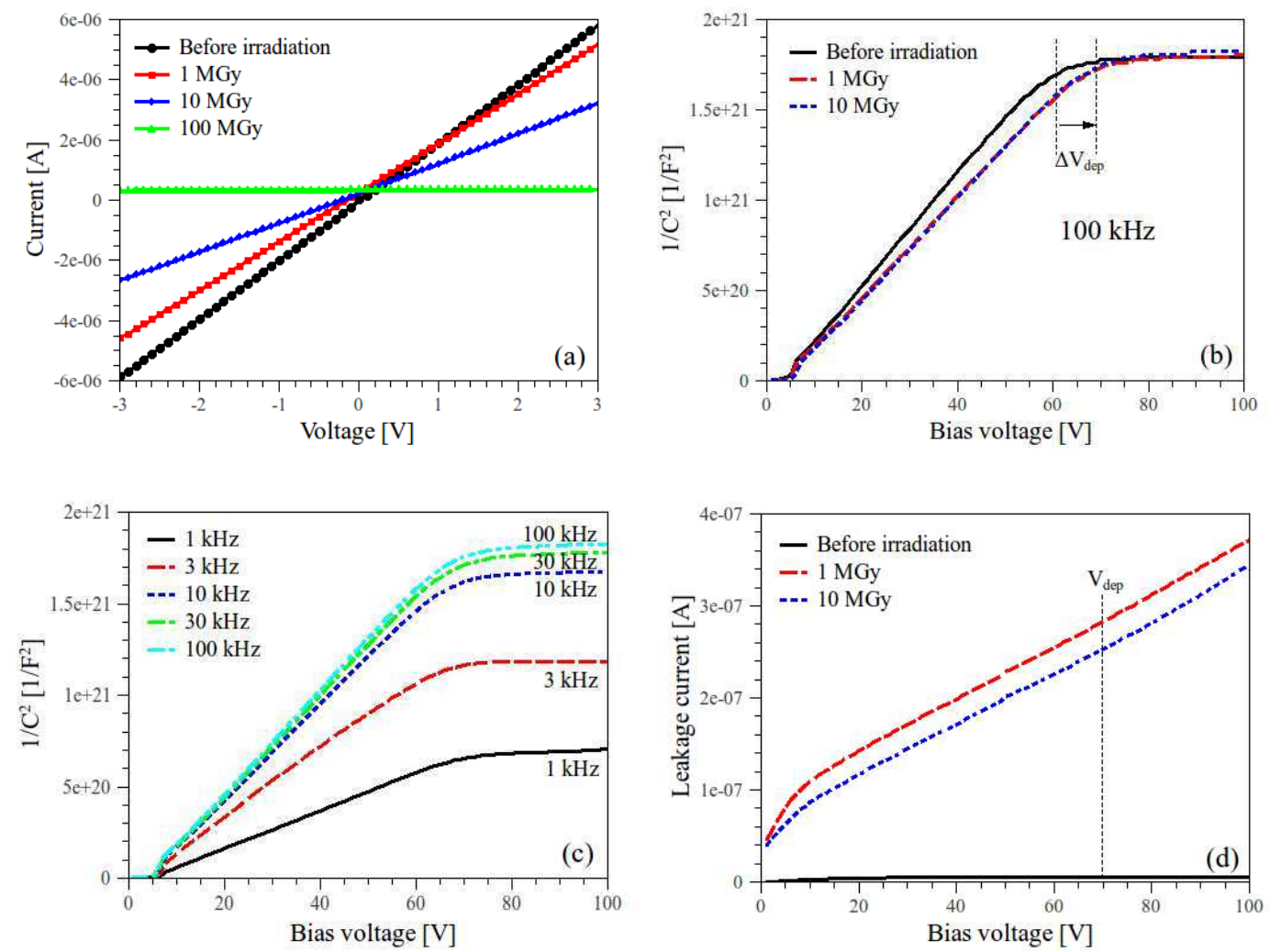

Figure 5. (a) I-V characteristics of a single biasing resistor. (b) The capacitance of the entire sensor at 100 $\mathrm{kHz}$, shown as $1 / \mathrm{C}^{2}$ versus bias voltage: Solid - 0 MGy; dashed - $1 \mathrm{MGy}$; dotted - $10 \mathrm{MGy}$. The data for 1 and 10 MGy are practically indistinguishable. (c) Sensor capacitance as function of bias voltage for 10 MGy dose. (d) Sensor leakage current: Solid - 0 MGy; dashed - 1 MGy; dotted - 10 MGy.

\subsection{Charge losses}

We have studied time resolved current pulses, produced by a sub-nano second laser pulse focused to $\sigma=3 \mu \mathrm{m}$, in the unirradiated and irradiated AC-coupled microstrip sensors as function of the position between the $\mathrm{p}^{+}$implants. The penetration of the $660 \mathrm{~nm}$ laser light in silicon is about $3 \mu \mathrm{m}$. It was found that the measured transient current and the integrated charges collected by the electrodes strongly depend on the irradiation dose of X-rays, biasing history, humidity and time. The shape of the transient signals and the integrated charge as function of the position of the light spot can be described by a model considering losses of either electrons or holes in the "weak" electric field region close to the $\mathrm{Si}-\mathrm{SiO}_{2}$ interface. A further publication which describes the method, presents the results on charge losses and discusses the relevance for X-ray doses is in preparation. 


\section{Summary and outlook}

MOS capacitors and AC-coupled microstrip sensors built on high resistivity <100> n-type silicon have been irradiated with $12 \mathrm{keV}$ X-rays to doses up to $1 \mathrm{GGy}$ and $100 \mathrm{MGy}$, respectively. For the MOS capacitors results on the fixed oxide charge density and interface trap density as function of dose and the procedure to extract the microscopic radiation damage parameters have been presented. It was found that at least three dominant interface traps have to be used to describe the measurements and to obtain a good description of the C/G-V curves. For doses above 10 - 100 MGy the densities of the oxide charges and of the three interface traps saturate. For the AC-coupled microstrip sensors, it was found that the change of the electrical properties, e.g. capacitance, leakage current and interstrip capacitance and resistance, and charge collection after irradiation are related to the width of the electron accumulation layer formed close to the $\mathrm{Si}_{-} \mathrm{SiO}_{2}$ interface. Its size increases with dose and decreases with applied bias voltage. It also depends on the biasing history, time and environmental conditions like humidity and temperature. The extracted parameters have been implemented into the Synopsys TCAD simulation program. They provide a reasonable description of the observations on silicon microstrip sensors.

\section{Acknowledgments}

The work was performed in the framework of the project "Radiation Damage" financed by the European XFEL-GmbH in close collaboration with the AGIPD project. J. Zhang would like to thank the Marie Curie Initial Training Network "MC-PAD" for financial support. We thank the crew of the HASYLAB DORIS III beam lines and colleagues within AGIPD collaboration for their help with setting up the irradiation facility and discussing the results. The work was also supported by the Helmholtz Alliance "Physics at the Terascale".

\section{References}

[1] A. Akkerman et al., Updated NIEL calculations for estimating the damage induced by particles and gamma-rays in Si and GaAs, Radiat. Phys. Chem. 62 (2001) 301.

[2] D. M. Fleetwood, M. R. Shaneyfelt and J. R. Schwank, Estimating Oxide-Trap, Interface-Trap, and Border-Trap Charge Densities in MOS Transistors, Appl. Phys. Lett. 64 (1994) 1965.

[3] H. Perrey, Jets at Low $Q^{2}$ at HERA and Radiation Damage Studies for Silicon Sensors for the XFEL, $\mathrm{PhD}$ thesis, Hamburg University, DESY-THESIS-2011-021 (2011) 153-186.

[4] A. Dasgupta et al., Dose Enhancement and Reduction in $\mathrm{SiO}_{2}$ and High- $\kappa$ MOS Insulator, IEEE Trans. Nucl. Sci. 57 (2010) 3463.

[5] J. Zhang et al., Study of radiation damage induced by $12 \mathrm{keV} \mathrm{X-rays} \mathrm{in} \mathrm{MOS} \mathrm{structures} \mathrm{built} \mathrm{on} \mathrm{high}$ resistivity n-type silicon, submitted to J. Synchrotron Rad., arXiv 1107.5949.

[6] J. G. Simmons and G. W. Taylor, High-Field Isothermal Currents and Thermally Stimulated Currents in Insulators Having Discrete Trapping Levels, Phys. Rev. B 5 (1972) 1619.

[7] H. A. Mar and J. G. Simmons, Determination of the energy distribution of interface traps in MIS system using non-steady-state techniques, Solid-State Electronics 17 (1974) 131. 
[8] H. A. Mar and J. G. Simmons, Surface-generation statistics and associated currents in metal-oxide-semiconductor structures, Phys. Rev. B 11 (1973) 775.

[9] E. H. Nicollian and J. R. Brews, MOS (Metal Oxide Semiconductor) Physics and Technology, John Wiley and Sons, Inc., New York 1982.

[10] J. Schwandt, Optimization of Silicon Pixel Sensors for the high X-ray Doses of the European XFEL, The 9-th International Conference on Position Sensitive Detectors, 12-16 September 2011, Aberystwyth University, United Kingdom, paper in preparation. 